$\underline{\xi}=$ 줄

\title{
Green tea in medicine: a brief overview
}

\author{
Abhishek Singh Nayyar ${ }^{1 *}$, Maneesha Das ${ }^{2}$, Bharat Deosarkar ${ }^{3}$, Soniya Bharat Deosarkar ${ }^{4}$, \\ Abhishek Karan ${ }^{5}$, Pallavi Sinha ${ }^{6}$, Swati Paraye ${ }^{7}$ \\ ${ }^{1}$ Reader cum PG Guide, Department of Oral Medicine and Radiology, Saraswati Dhanwantari Dental College \\ and Hospital and Post-Graduate Research Institute, Parbhani, Maharashtra, India \\ ${ }^{2}$ Reader, Department of Conservative Dentistry and Endodontics, Saraswati Dhanwantari Dental College \\ and Hospital and Post-Graduate Research Institute, Parbhani, Maharashtra, India \\ ${ }^{3}$ Senior Lecturer, Department of Conservative Dentistry and Endodontics, Saraswati Dhanwantari Dental College \\ and Hospital and Post-Graduate Research Institute, Parbhani, Maharashtra, India \\ ${ }^{4}$ Senior Lecturer, Department of Prosthodontics and Crown and Bridge, Saraswati Dhanwantari Dental College \\ and Hospital and Post-Graduate Research Institute, Parbhani, Maharashtra, India \\ ${ }^{5}$ Senior Lecturer, Department of Oral and Maxillofacial Surgery, Saraswati Dhanwantari Dental College \\ and Hospital and Post-Graduate Research Institute, Parbhani, Maharashtra, India \\ ${ }^{6}$ Senior Lecturer, Department of Oral Medicine and Radiology, Saraswati Dhanwantari Dental College \\ and Hospital and Post-Graduate Research Institute, Parbhani, Maharashtra, India \\ ${ }^{7}$ Senior Lecturer, Department of Oral Medicine and Radiology, Saraswati Dhanwantari Dental College \\ and Hospital and Post-Graduate Research Institute, Parbhani, Maharashtra, India \\ *Corresponding author E-mail: singhabhishekndls@gmail.com
}

\begin{abstract}
Over the past decades, focused interest in drugs obtained from medicinal plants has markedly increased. Since times immemorial, tea has traditionally been the most popular and widely consumed beverages in the world obtained from the extracts of leaves of the plant Camellia sinensis by the process of oxidation. It is the oldest non-alcoholic beverage containing caffeine. Among the varieties of tea manufactured, green tea is considered of utmost significance since it exerts antimicrobial, anti-cancer, antioxidant, anti-collagenase, anti-diabetic, anti-obesity and anti-hypertensive activities due to rich content of polyphenol, namely catechins, which prevent the pathogenesis of numerous disease processes and play a pivotal role as a scavenger of free radical generation. The present review discusses the botanical description, photo-chemical constituents and biological activity of green tea with clinical relevance in the various fields of medicine.
\end{abstract}

Keywords: Green Tea; Camellia Sinensis; Antimicrobial; Anti-Cancer; Antioxidant; Anti-Collagenase; Anti-Diabetic; Anti-Obesity; Anti-Hypertensive; Polyphenol; Photo-Chemicals

\section{Introduction}

Since ancient civilizations, natural plants have been used as traditional medicines for the treatment of various ailments. Alternative medicines are a part of primary health care regimens due to highcost treatment regimens and cross-resistance to chemically manufactured drugs. The affordability and safety issues have drawn the world towards the use of photo-medicines to treat various human diseases. Tea is the second most widely consumed beverage ever since 3000 B.C. (Mahmood T et al. 2010) it is obtained from infusion of the extracts of leaves and leaf buds of plant Camellia sinensis originally from South West China. It was originally used as a medicine for various illnesses as a bitter concoction since they were rich in polyphenol catechins. (Cabrera C et al. 2006) According to the different methods of processing, three types of tea are produced including Black Tea (fully oxidized/fermented), Oolong Tea (semi-oxidized/ fermented) and Green Tea (Nonoxidized/non-fermented). Green tea is the most important amongst all since it contains the highest amount of polyphenol catechins. Catechins are dietary polyphenolic compounds associated with a wide variety of beneficial health effects proven in-vitro, in-vivo and clinically.
Emerging evidence has shown catechins and their metabolites to possess antimicrobial, antioxidant, anti-collagenase, anti-cancer, anti-diabetic, anti-obesity, anti-hypertensive and anti-asthmatic activities preventing pathogenesis of numerous disease processes and playing a pivotal role as a scavenger of free radical generation. (Narotzki B et al. 2012) So, researchers are in a constant look-out for alternatives to the medicines used in the treatment of infections due to their varied adverse effect profiles and development of resistant strains. (Chacko SM et al. 2010, Chan EWC et al. 2011).

The present review discusses the botanical description, phytochemical constituents and biological activity of green tea with clinical relevance in the various fields of medicine.

\section{Pharmacological uses of green tea in vari- ous aspects of medicine}

Green tea has an important role on various molecular targets such as tumor necrosis factor-alfa (TNF-alfa) and interleukin-2 (IL-2), thus, suggesting that a polyphenolic extracted of green tea, epiglottectomy-3-gallate (EGCG), could modulate various biochemical pathways involved in the inflammatory responses and joint

Copyright $\odot 2017$ Dr.Abhishek Singh Nayyar et al. This is an open access article distributed under the Creative Commons Attribution License, which permits unrestricted use, distribution, and reproduction in any medium, provided the original work is properly cited. 
destruction seen in arthritis. EGCG inhibits interleukin-1 beta (IL1 beta) induced cartilage proteoglycan degradation and expression of enzymes matrix metalloproteins-1 and 13 (MMP-1 and 13) in human chondrocytes, thus, suppressing precipitation of cartilage degradation seen in arthritis. (Katiyar SK \& Raman C 2011) EGCG catechin mainly inhibits alpha amylase activity in saliva as well as decreases intestinal amylase activity, thus, lowering the hydrolysis of starch and glycogen to glucose reducing glucose assimilation and hence, decreasing the digestive enzyme activity. It, also, increase's tyrosine phosphorylation of insulin receptor, insulin receptor substrate and reduces gene expression of gluconeogenic enzymes, namely phosphoenolpyruvate carboxykinase, thereby, exerting an anti-diabetic action.

Polyphenol in green tea protect the body against oxidative stress associated with late complications of diabetes. Regular consumption of green tea reduces body weight and suppresses the dietary food intake. EGCG has an inhibitory effect on enzyme acetyl carboxylase, which has an important role in the fatty acid biosynthesis. It influences sympathetic nervous system activity, up regulates enzymes involved in hepatic fat oxidation and increases fat oxidation within the body, thus, preventing fat accumulation.

Caffeine along with catechins present act synergistically to increase fat oxidation within the body and prolong sympathetic stimulation of thermogenesis. (Watanabe J et al. 1998, Sabu MC et al. 2002) It, also, prevents osteoporosis commonly seen in elderly females. L-theanine present in green tea has been proven to lower high arterial blood pressure and is a good anti-hypertensive agent. (Yokogoshi $\mathrm{H}$ et al. 1995, Hegarty VM et al. 2000)

Green tea improves the immune status of the body through antioxidant activity and cytokine gene expression in cases of immunocompromised patients like heavy smokers. The heat-protective role of green tea on cadmium-induced heat-toxicity in liver has been evaluated, and it has been shown that green tea shows decreased expression of hepatic enzymes, including serum glutamate pyruvate transaminase (SGPT), serum glutamate oxaloacetate transaminase (SGOT), gamma-glutamyl transferase (GGT) and lactate dehydrogenase (LDH). (El-Beshbishy HA 2005, Kuo CL et al. 2014).

Green tea constituents like catechins and theanine have neuroprotective activities by minimizing eicosanoid accumulation and oxidative damage in cases of ischemia-induced brain injury. EGCG improves age-related cognitive-deficits and protects against cerebral ischemia. It has, also, been shown to exert the protective effect against beta-amyloid-induced neuro-toxicity in cultured hippocampal neurons as observed in the various animal models. Green tea has anti-stress, calming and healing properties due to the presence of L-theanine which is believed to facilitate the generation of alpha waves in the brain and reduce cortisol levels during stress to produce relaxed yet alert state of mind.

Green tea, also, possesses a potent anti-asthmatic activity via an increased expression of tumour necrosis factor-beta (TNF-beta), interferon-gamma (IFN-gamma) and decreasing anti-asthmatic cytokines in the lungs. (Kakuda T 2002, Heo JC et al. 2007).

Consumption of green tea substantially decreases risk of cardiovascular diseases and prevents atherogenesis by protecting lowdensity lipoproteins (LDL) fraction against oxidative injuries. There is activated synthesis of prostaglandins (PGs) and catechins have anti-platelet and metal chelating properties. $\alpha$-A-tocopherol which is the main antioxidant to protect LDL against oxidative injuries is regenerated due to green tea consumption. EGCG reduces cholesterol and triacylglycerol levels in the blood by interfering with the micellar solubilization of cholesterol in the digestive tract and its absorption.

Green tea extracts have inhibitory effect on cyclo-oxygenase (COX) and lipoxygenase (LOX) pathways of arachidonic acid metabolism. Antioxidants present in green tea neutralize the adverse effects of free radicals in the body and reduces DNA damage, thus, protecting the skin from the harmful UV rays. (Elmets CA et al. 2001, Hirano R et al. 2002).
Green tea polyphenol, also, prevent diabetic retinopathy by modulation of genetic pathways such as angiogenesis and by antioxidant defense mechanism.

Green tea has shown antimicrobial activity against enteric pathogens like S. epidermis, Methicillin-resistant Staphylococcus aureus (MRSA), Methicillin-sensitive Staphylococcus aureus, Helicobacter pylori (H. pylori), Staphylococcus aureus, Streptococcus mutans, Salmonella typhi, Shigella sp. and Vibrio cholera. EGCG has been found to inhibit influenza virus $\mathrm{A}$ and $\mathrm{B}$, HIV virus enzymes, Hepatitis B, Rota virus and Enteroviruses in monkey cell cultures. EGCG binds to the cell-surface CD4 molecules and prevents HIV infection throughout the body. It has, also, been found to be effective at a wide range of fungi, namely Candida albicans, Candida glabrata, Candida kruseii, Candida dubliensis. (Horiba N et al. 1991, Kumar B et al. 2012).

\section{Anti-cancer potential of green tea and mechanisms proposed}

Various experimental reports have shown green tea to possess antioxidant potential due to rich content of catechins and tannins. Reduced free radical generation, lipid peroxidation and oxidative DNA damage had been reported after green tea consumption in smokers. Green tea has anti-cancer effects via modulation of genes involved in cancer initiation, promotion and progression acting in the following manner: (Suganuma M et al. 2011, Alappat B et al. 2015, Ullah $\mathrm{N}$ et al. 2016)

1) It acts as an inhibitor of cyclo-oxygenase (COX) and lipoxygenase (LOX) pathways of arachidonic acid metabolism and tumour necrosis factor (TNF) and interleukin (IL) pathways for development and progression of cancer cells;

2) It has preventive effect via activation of tumour suppressor genes such as p53 and pTEN/p21, inhibits angiogenesis by suppressing activation of HIF- $1 \alpha$ and NF- $\kappa B$ pathways, regulates apoptosis and prevents other transcription factors which are involved in the development and progression of cancer cell lines;

3) It has antioxidant potential and neutralises free radicals, thus, preventing progress of the tumour in the body;

4) It modulates the genes involved in the process of carcinogenesis. Green tea polyphenols and EGCG activates p53 gene by increasing its transcriptional activity and acetylation. EGCG inhibits growth and invasion of cancer cells by causing cell cycle arrest and inducing apoptosis in them;

5) It inhibits growth of cancer cells by inhibiting activation of oncogenes such as HER2 and HER3 and epidermal growth factor (EGF) receptors;

6) It causes inhibition of telomerase, AP-1 and IGFIR responsible for carcinogenesis;

7) It activates peroxisome-proliferator receptors inducing apoptosis in various cancer cell lines.

EGCG present in green tea has been shown to suppress the growth of melanoma cells in-vivo and has been found to be effective in chronic lymphatic leukemia (CLL) patients in Phase 1 trials. It is a potent chemotherapeutic agent causing G1 cell cycle arrest and inducing apoptosis in such patients. (Khan N et al. 2006, Call TG et al. 2009) EGCG and ECG significantly reduce interleukin-6 and 8 (IL-6 and 8) expression in the cells exposed to prostaglandins (PGs). Intercellular adhesion molecule-1 and vascular adhesion molecule-1 expression is, also, reduced after treatment with EGCG and ECG. (Suyama E et al. 2011, Chaterjee P et al. 2012).

\section{Green tea in bone resorptions}

Green tea intake leads to the inhibition of enzymes matrix metalloproteinases (MMPs). (Sakanaka S \& Okada Y 2004) EGCG has, also, been shown to reduce the size of bone defects by inhibiting the expression of enzyme cysteine in osteoblasts and decreasing the infiltration of macrophages preventing bone resorptions. (Lee 
YL et al. 2009) EGCG, also, has been reported to have the potential to inhibit the activity of enzyme proteinases. Matrix metalloproteinases-1, 2, 9 and 13 (MMP-1, 2, 9 and 13) are the main factors involved in carrying-out the resorption of bone collagen by osteoblasts. Green tea polyphenols, also, protect the bone from resorption due to inhibition of the enzyme matrix metalloproteinase-9 (MMP-9) in osteoblasts and activation of osteoclasts. (Yun JH et al. 2004).

\section{Green tea: Negative effects of a positive, healthy drink}

Although green tea has been proven to have therapeutic benefits in the various disease set-ups, excessive intake of green tea can be harmful to the body and is known to cause numerous adverse effects including: (Grover HS et al. 2015).

1) It blocks the absorption of nutrients like iron and thiamine;

2) It might lead to symptoms of restlessness, irritability, palpitations, loss of appetite, nausea, gastro-intestinal upsets and rashes;

3) It can result in easy and prolonged bleeding tendencies due to increase in bleeding time;

4) EGCG present in green tea extracts is cytotoxic, if consumed, in larger quantities;

5) Caffeine and catechins present in green tea extracts can cause birth defects due to folic acid antagonism;

6) Excessive intake of green tea in pregnant and breast feeding females can cause palpitations due to the presence of vasoconstrictive and stimulative caffeine;

7) Excessive intake of green tea accumulates aluminium in the body resulting in various neurological disorders.

\section{Discussion}

The present-day sedentary lifestyle and dietary habits have increased the risk for a plethora of diseases, including various cancers and cardiovascular disorders. Use of phyto-medicine in the treatment of such diseases has considerably increased in the recent years owing to the potential toxic effects of the conventional therapeutic agents used. Tea is considered as a pleasant, economic and socially accepted health beverage proving to be a potent phytotherapeutic agent in the prevention of chronic diseases in humans. Various epidemiological studies and laboratory investigations have indicated that polyphenols, namely catechins present in the green tea possess strong antimicrobial, anti-cancer, antioxidant, anti-collagenase, anti-diabetic, anti-obesity and anti-hypertensive activities, which prevent the pathogenesis of numerous disease processes and play a pivotal role as a scavenger of free radical generation. (Sabu MC et al. 2002, Chacko SM et al. 2010, Chan EWC et al. 2011, Katiyar SK \& Raman C 2011) These polyphenols, also, promotes other physiological functions, including antifibrotic and neuro-protective properties and protects skin from the harmful UV radiations. They, also, contribute to increase the bone density and improve body immunity. (Watanabe J et al. 1998, Hegarty VM et al. 2000, Kakuda T 2002, Kuo CL et al. 2014) Several mechanisms have been presented to explain the chemopreventive effects of EGCG amongst which its effect to target specific cell signaling pathways have received considerable attention for regulating the cellular proliferation and the process of apoptosis. The diversified effects of EGCG include its broad pharmacological effects in modulating the cell signaling pathways. In-vivo studies have proven that green tea catechins increase the total plasma antioxidant activity by decreasing the production of malondialdehyde, which is a marker of oxidative stress. Green tea has been proven to have rich antioxidant properties and better free radical scavenging property than Vitamin $\mathrm{C}$ and $\mathrm{E}$. In addition to other mechanisms, it is known to activate cell death signals and induce apoptosis in pre-cancerous and cancer cell lines resulting in the inhibition of the development and progression of cancer cells
EGCG modulates the signal transduction pathways involved in cell proliferation, transformation, apoptosis and metastasis. Most importantly, these anti-proliferative and pro-apoptotic effects of EGCG have been shown to be selective for cancer cells only as normal cells are not affected during the treatment procedure. In cancer cells, EGCG, also, causes inhibition on the activity of specific receptor tyrosine kinases and related downstream pathways of signal transduction. Hence, it acts synergistically to enhance the effect of various anti-cancer drugs when used in combination therapy. (Khan N et al. 2006, Call TG et al. 2009, Chaterjee P et al. 2012) Numerous studies have revealed the antibacterial effect of green tea by the stimulation of protective components such as immunoglobulins, lysosomes, lactoferrin, histatins and mucin. Polyphenols, also, reinforce to increase the collagen formation and accelerate the soluble-to-insoluble collagen conversion during tissue regeneration. They, also, prohibit the production of PGE2 and can, thus, reduce the progress through the process of bone resorption. (Suyama E et al. 2011) Though with potential advantages and therapeutic benefits, information regarding the bioavailability of catechins and other polyphenols present in green tea extracts is limited in humans studies. Further studies are, therefore, mandated to know the exact details about their absorption, distribution and metabolism in the body. Hence, a careful evaluation of the available data is mandatory to harness the complete therapeutic advantages they can offer for improving the health outcomes without significantly compromising the body systems with the common adverse effects seen with the conventional therapeutic agents. (Awadalla HI et al. 2011, Nugala B et al. 2012)

\section{Conclusion and future research directions}

Phyto-medicine has been of increasing interest in the recent years. The major advantages of green tea are its safety, easy availability, cost-effectiveness, increased shelf life and a lack of microbial resistance. According to the substantial evidence available, green tea has been proven to have potential therapeutic properties. However, the literature is scarce with regard to the information about the quality, safety and efficacy of its potential use in various disease set-ups. Hence, further clinical trials are mandated to support its use as effective alternatives to the synthetically available drugs. Furthermore, research is, also, mandated to investigate into the toxicity and possible drug interactions of their constituents in various aspects of medicine.

\section{References}

[1] Mahmood T, Akhtar N, Khan BA (2010). The morphology, characteristics and medicinal properties of Camellia sinensis tea. $\mathrm{J}$ Med Plants Res 4, 2028-33. https://doi.org/10.5897/JMPR10.010.

[2] Cabrera C, Artacho R, Gimenez R (2006). Beneficial effects of green tea: $A$ review. $\mathrm{J}$ Am Coll Nutr 25, 79-99. https://doi.org/10.1080/07315724.2006.10719518.

[3] Narotzki B, Reznick AZ, Aizenbud D, Levy Y (2012). Green tea: A promising natural product in oral health. Arch Oral Biol 57, 429-35. https://doi.org/10.1016/j.archoralbio.2011.11.017.

[4] Chacko SM, Thambi PT, Kuttan R, Nishigaki I (2010). Beneficial effects of green tea: A literature review. Chinese Med 5, 1-9. https://doi.org/10.1186/1749-8546-5-13.

[5] Chan EWC, Soh EY, Tie PP, Law YP (2011). Antioxidant and antibacterial properties of green, black and herbal teas of Camellia sinensis. Pharmacognosy Res 3, 266-72. https://doi.org/10.4103/0974-8490.89748.

[6] Katiyar SK, Raman C (2011). Green tea: A new option for the prevention or, control of osteoarthritis. Arthritis Res Ther 13, 121. https://doi.org/10.1186/ar3428.

[7] Watanabe J, Kawabata J, Niki R (1998). Isolation and identification of acetyl-CoA carboxylase inhibitors from green tea (Camellia sinensis). Biosci Biotechnol Biochem 62, 532-4. https://doi.org/10.1271/bbb.62.532.

[8] Sabu MC, Smitha K, Kuttan R (2002). Anti-diabetic activity of green tea polyphenols and their role in reducing oxidative stress in experimental diabetes. J Ethnopharmacol 83, 109-16. https://doi.org/10.1016/S0378-8741(02)00217-9. 
[9] Yokogoshi H, Kato Y, Sagesaka YM, Matsuura T (1995). Reduction effect of theanine on blood pressure and brain 5hydroxyindoles in spontaneously hypertensive rats. Biosci Biotechnol Biochem 59, 615-8. https://doi.org/10.1271/bbb.59.615.

[10] Hegarty VM May HM, Khaw KT (2000). Tea drinking and bone mineral density in older women. Am J Clin Nutr 71, 1003-7.

[11] El-Beshbishy HA (2005). Hepato-protective effect of green tea (Camellia sinensis) extract against tamoxifen-induced liver injury in rats. J Biochem Mol Biol 38, 563-70.

[12] Kuo CL, Chen TS, Liou SY, Hsieh CC (2014). Immunomodulatory effects of EGCG fraction of green tea extract in innate and adaptive immunity via $\mathrm{T}$ regulatory cells in murine model. Immuno-pharmacol Immuno-toxicol 36, 364-70. https://doi.org/10.3109/08923973.2014.953637.

[13] Kakuda T (2002). Neuro-protective effects of the green tea components theanine and catechins. Biol Pharm Bull 25, 1513-8. https://doi.org/10.1248/bpb.25.1513.

[14] Heo JC, Kim SH, Kim TH, Lee SH (2007). An aqueous extract of green tea Camellia sinensis increases expression of TH1 cell specific anti-asthmatic markers. Int J Mol Med 22, 763-7.

[15] Elmets CA, Singh D, Tubesing K, Matsui M, Katiyar S, Mukhtar H (2001). Cutaneous photo-protection from ultraviolet injury by green tea polyphenols. J Am Acad Dermatol 44, 425-32. https://doi.org/10.1067/mjd.2001.112919.

[16] Hirano R, Momiyama Y, Takahashi R, Taniguchi H, Kondo K, Nakamura $\mathrm{H}$, et al (2002). Comparison of green tea intake in Japanese patients with and without angiographic coronary artery disease. Am J Cardiol 90, 1150-3. https://doi.org/10.1016/S0002 9149(02)02787-X

[17] Horiba N, Maekawa Y, Ito M, Matsumoto T, Nakamura H (1991). A pilot study of Japanese green tea as a medicament with antibacterial and bactericidal effects. $J$ Endod 17, 122-4 https://doi.org/10.1016/S0099-2399(06)81743-7.

[18] Kumar B, Gupta SK, Nag TC, Srivastava S, Saxena R (2012) Green tea prevents hyperglycemia-induced retinal oxidative stress and inflammation in streptozotocin-induced diabetic rats. Opthalmic Res 47, 103-8. https://doi.org/10.1159/000330051.

[19] Suganuma M, Saha A, Fujiki H (2011). New cancer treatment strategy using combination of green tea catechins and anti-cancer drugs. J Japanese Cancer Assoc 102, 317-23. https://doi.org/10.1111/j.1349-7006.2010.01805.x.

[20] Alappat B, Jaclyn A, Truong SC (2015). Anti-cancer and Antioxidant Properties of Flavoured Green Tea Extracts. J of Agriculture and Life Sciences 2, 15-24.

[21] Ullah N, Ahmad M, Aslam H, Tahir MA, Aftab M, Bibi N, et al (2016). Green tea phyto-compounds as anti-cancer: A review. Asian Pac J Trop Dis 6, 330-6. https://doi.org/10.1016/S2222 1808(15)61040-4

[22] Khan N, Afaq F, Saleem M, Ahmad N, Mukhtar H (2006). Targeting Multiple Signalling Pathways by Green Tea PolyphenolEpigallo-catechin-3-Gallate. Cancer Res 66, 2500-5. https://doi.org/10.1158/0008-5472.CAN-05-3636.

[23] Call TG, Shanafelt TD (2009). Phase I trial of daily oral polyphenol in patients with asymptomatic stage of chronic lymphocytic leukemia. J Clin Oncol 27, 3808-14 https://doi.org/10.1200/JCO.2008.21.1284.

[24] Suyama E, Tamura T, Ozawa T, Suzuki A, Iijima Y, Saito T (2011) Re-mineralization and acid resistance of enamel lesions after chewing gum containing fluoride extracted from green tea. Aust Dent $\mathrm{J}$ 56, 394-400. https://doi.org/10.1111/j.1834-7819.2011.01359.x.

[25] Chaterjee P, Chandra S, Dey P, Bhattacharya S (2012). Evaluation of anti-inflammatory effects of green tea and black tea: A comparative in-vitro study. J Adv Pharm Technol Res 3, 136-8. https://doi.org/10.4103/2231-4040.97298.

[26] Sakanaka S, Okada Y (2004). Inhibitory effects of green tea polyphenols on the production of a virulence factor of the periodontal disease causing anaerobic bacterium Porphyromonas gingivalis. J Agric Food Chem 52, 1688-92. https://doi.org/10.1021/jf0302815.

[27] Lee YL, Hong CY, Kok SH, Hou KL, Lin YT, Chen MH (2009). An extract of green tea, Epigallocatechin-3-gallate reduces periapical lesions by inhibiting cysteine-rich 61 expression in osteoblasts. J Endod 35, 206-11. https://doi.org/10.1016/j.joen.2008.11.015

[28] Yun JH, Pang EK, Kim CS, Yoo YJ, Cho KS, Chai JK (2004). Inhibitory effects of green tea polyphenol epigallo-catechin-gallate on the expression of matrix metalloproteinase- 9 on the formation of osteoclasts. J Periodont Res 39, 300-7. https://doi.org/10.1111/j.1600-0765.2004.00743.x.
[29] Grover HS, Deswal H, Bharadwaj A (2015). Green tea as an alternative therapy in medicine and dentistry: A review. Int Ayurved Med J 3, 516-23.

[30] Awadalla HI, Ragab MH, Bassuoni MW, Fayed MT (2011). A pilot study of the role of green tea use on oral health. Int J Dent Hyg 9, 110-6. https://doi.org/10.1111/i.1601-5037.2009.00440.x.

[31] Nugala B, Namasi A, Emmadi P, Krishna PM (2012). Role of green tea as an antioxidant in periodontal disease: The Asian paradox. J Indian Soc Periodontol 16, 313-6. https://doi.org/10.4103/0972124X.100902. 